IMECE2008-68079

\title{
ELECTRICAL LEAKAGE THROUGH THIN PDMS MICROCHANNEL WALLS AND ITS APPLICATIONS
}

\author{
Jiashu Sun ${ }^{1}$ \\ Saumitra K. Vajandar ${ }^{1}$ \\ Dongyan $\mathrm{Xu}^{1}$ \\ Yuejun Kang ${ }^{1}$ \\ Dongqing $\mathrm{Li}^{1}$ \\ Deyu $\mathrm{Li}^{1}$ \\ Guoqing $\mathrm{Hu}^{2}$ \\ ${ }^{1}$ Department of Mechanical Engineering, Vanderbilt University, Nashville, TN 37235 \\ ${ }^{2}$ Institute of Mechanics, Chinese Academy of Science, Beijing 100080, China
}

\section{ABSTRACT}

PDMS is usually considered as a dielectric material and PDMS microchannel walls can be treated as an electrically insulated boundary. However, in certain layouts of microfluidic networks, electrical leakage through PDMS walls could significantly alter the electrical field in the microfluidic circuits, which must be carefully considered in microfluidic circuit design. We report on our experimental characterization of electrical leakage through PDMS microfluidic channel walls. Numerical modeling clearly disclosed the alteration of electrical field and electroosmotic velocity in the microfluidic channels because of the electrical leakage through the thin PDMS wall. In addition, we demonstrate that the electrical leakage through the PDMS channel wall can be used to realize trapping of individual particles at different locations inside the mcirofluidic channel by balancing the electroosmotic flow and electrophoretic migration of the particle.

\section{INTRODUCTION}

Poly(dimethylsiloxane) (PDMS), which is elastic, optically transparent, nontoxic, and biologically compatible, has been extensively used in lab-on-a-chip devices because they can be easily fabricated by soft lithography techniques at low cost (McDonald et al. 2002). As more compact and complex microfluidic circuits are being developed, electrical leakage through small PDMS structures has attracted more attention because the leakage might totally alter the device performance.

\section{LEAKAGE CURRENT CHARACTERIZATION}

Figure 1 shows the basic structure of the chip design for measuring the leakage current. In this experiment, there are four different configurations of a main (center) channel (100 $\mu \mathrm{m}$ wide and $18000 \mu \mathrm{m}$ long) sandwiched between two side channels, which are symmetric and separated from the main channel by a $15 \mu \mathrm{m}$ thick PDMS channel wall. Three of the four configurations vary only in dimension, while the fourth configuration has a different structure (Fig. 2), which is also used for the particle trapping experiment later.

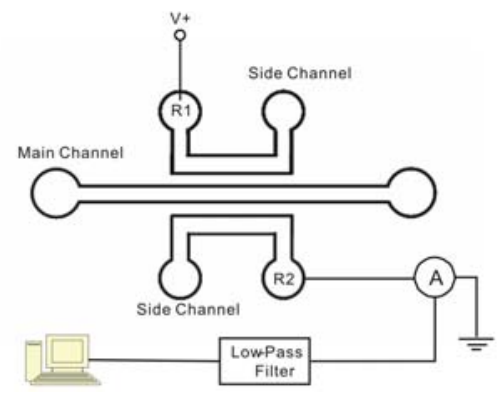

Fig. 1 Schematic of leakage current measurement in the PDMS-based microfluidic device.

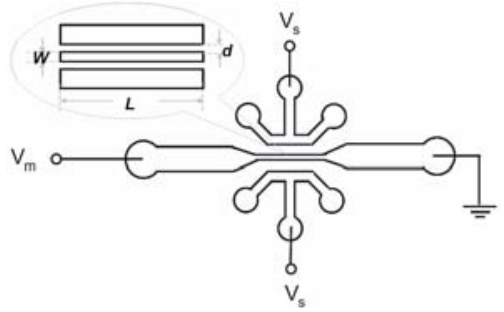

Fig. 2 Schematic of microfluidic chips for testing the leakage current and for particle trapping. In the center region, the width of the main channel shrinks from $100 \mu \mathrm{m}$ to $15 \mu \mathrm{m}$, forming a particle trapping section which has a dimension of $2000 \times 15 \times 15 \mu \mathrm{m}^{3}$ (length $\times$ width $\times$ depth).

The microfluidic devices were filled with $10 \mathrm{mM}$ sodium borate buffer. A positive voltage was applied to the left-side well (R1) of the top side channel and the right-side well (R2) of the bottom side channel is grounded. The leakage current 
was measured with a current preamplifier (Model 1211, DL Instruments, NY), which was fed into the data acquisition system after being processed with a low-pass filter (Model SR560, Stanford Research Systems, CA). Fig. 3 shows the measured leakage current under applied voltages ranging from $10 \mathrm{~V}$ to $50 \mathrm{~V}$ for the four different side channels.
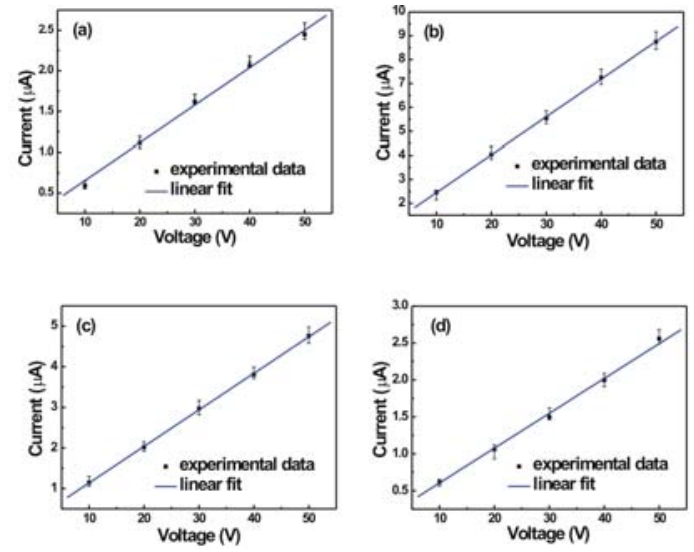

Fig. 3 Measured leaking current and the fitting curves of current versus voltage through PDMS channel walls for four different side channel geometries (length of horizontal section $\times$ width $\times$ depth): (a) $100 \times 100 \times 30 \mu \mathrm{m}^{3}$, (b) $500 \times 100 \times 30$ $\mu \mathrm{m}^{3}$, (c) $2000 \times 100 \times 15 \mu \mathrm{m}^{3}$, and (d) $2000 \times 100 \times 45 \mu \mathrm{m}^{3}$

In our calculation of the leakage conductivity of the PDMS channel wall, we take all the measured resistance as the resistance from the PDMS channel wall, which is a conservative estimation of the lowest leakage conductivity. Table 1 summarizes the electrical resistance and conductivity of the PDMS walls. Compared to the electrical conductivity of bulk PDMS $\left(2.5 \times 10^{-14} \mathrm{~S} / \mathrm{m}\right)$, the measured leakage electrical conductivity of the thin $(15 \mu \mathrm{m})$ PDMS structure in our devices is significantly larger (about $1.2 \times 10^{9} \sim 1.9 \times 10^{10}$ times of the bulk value). Note that the leakage conductivity can vary by up to one order of magnitude for different cases, which may reflect the variation in the fabrication process.

Table 1 Summary of measured electrical conductivity of small PDMS structures in the microfluidic devices

\begin{tabular}{ccc}
\hline Geometry (width/length/depth, $\mu m)$ & Resistance $(\Omega)$ & Conductivity $(s / m)$ \\
\hline $15 / 100 / 30$ & $2.14 \times 10^{7}$ & $4.67 \times 10^{-4}$ \\
$15 / 500 / 30$ & $6.33 \times 10^{6}$ & $3.16 \times 10^{-4}$ \\
$15 / 2000 / 45$ & $1.11 \times 10^{7}$ & $3.00 \times 10^{5}$ \\
$15 / 2000 / 15$ & $2.06 \times 10^{7}$ & $4.85 \times 10^{-5}$ \\
\hline
\end{tabular}

\section{TRAPPING OF INDIVIDUAL PARTICLES}

Based on the measured leakage current, we simulated the electric field in the main channel, side channels, and PDMS channel walls. The electric field is described by the Laplace equation with varied conductivities:

$$
\nabla \cdot\left(\sigma_{i} \nabla V\right)=0
$$

Where $\sigma_{i}$ represents the electrical conductivities of different regions in the simulation domain. According to the experimental measurement, the conductivity of $1 \mathrm{mM}$ sodium borate buffer is around $0.01 \mathrm{~S} / \mathrm{m}$, and the leakage conductivity of $15 \mu \mathrm{m}$ thick PDMS in the present device is around $3.0 \times 10^{-5}$ $\mathrm{S} / \mathrm{m}$. Furthermore, the electroosmotic flow in the main channel was also calculated by the Navier-Stokes equation:

$$
\rho \vec{u} \cdot \nabla \vec{u}=-\nabla P+\eta \nabla^{2} \vec{u},
$$

with the Helmholtz-Smoluchowski slip boundary condition at channel walls. The above equation is numerically solved using the finite element package Comsol Multiphysics 3.2 (Comsol Inc., MA) for the case of $5 \mathrm{~V}$ electrical bias across the main channel, and the simulation result is plotted in Fig. 4. Even though the overall potential drop across the channel does not change, the local electric field is significantly different, and hence the behavior of particles and fluid will be different.

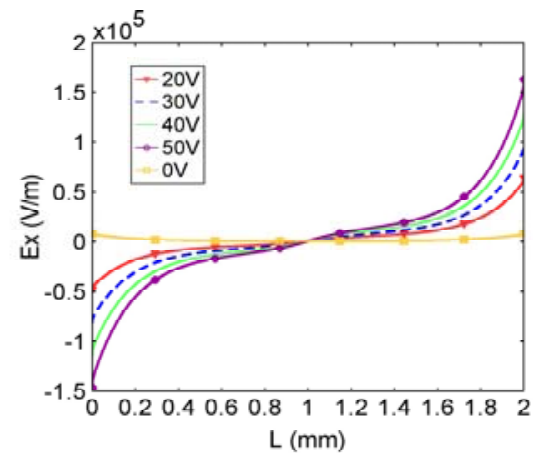

Fig. 4 Numerical predictions of the electric field strength under different positive gate voltages applied to the side channel.

Here we used $4 \mu \mathrm{m}$ (Bangs Laboratories, Inc, IN) in diameter beads to demonstrate trapping of individual particles. The microfluidic device for this purpose is filled with $1 \mathrm{mM}$ sodium borate buffer and shown schematically in Fig. 2. An electrical bias of $5 \mathrm{~V}$ was applied to the main channel to induce electroosmotic flow and introduce the particles into the main channel. Then different positive gate voltages (e.g. 20 $\mathrm{V}, 30 \mathrm{~V}$, and $50 \mathrm{~V}$ ) were applied to the middle reservoir of both side channels. As expected, we observed that the particles were trapped at different locations in the downstream section of the main channel. Fig. 5 shows the images of particle trapping under different applied positive gate voltages. We believe that the observed particle trapping is because the electroosmotic flow is balanced by the electrophoretic migration of the particle.

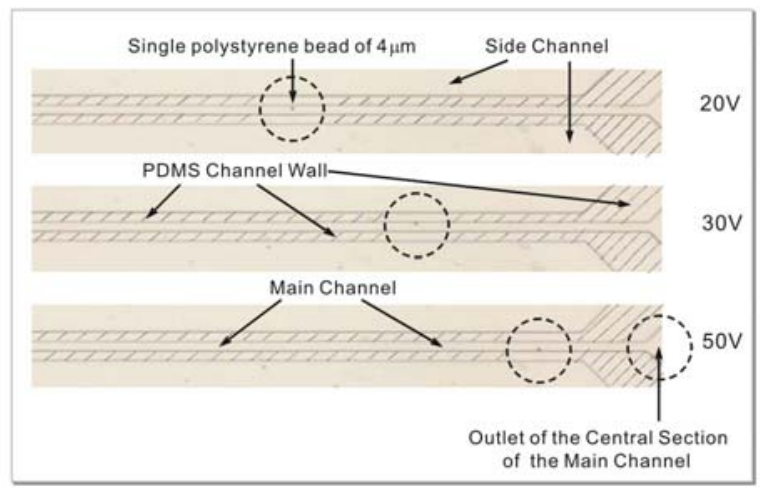

Fig. 5 Images of the microbead trapping using the electrical leakage. The dashed circle denotes the position where microbead is trapped.

\section{REFERENCE}

McDonald, J. C. and Whitesides, G. M., 2002, "Poly(dimethylsiloxane) as a material for fabricating microfluidic devices”, Acc. Chem. Res., 35:491-499. 